\title{
ON THE BACKWARD HEAT PROBLEM: EVALUATION OF THE NORM OF $\frac{\partial u}{\partial t}$
}

\author{
YVES BIOLLAY \\ Department of Mathematics \\ Corne11 University \\ Ithaca, New York 14850 U.S.A. \\ (Received July 19, 1979)
}

ABSTRACT. We show in this paper that $\|\Delta u\|=\left\|u_{t}\right\|$ is bounded $\forall t \leq T{ }^{(0)}<T$ if one imposes on $u$ (solution of the backward heat equation) the condition $\|u(x, t)\| \leq M$. A Hölder type of inequality is also given if one supposes $\left\|u_{t}(x, T)\right\| \leq K$.

KEY WORDS AND PHRASES: Parabolic equations, Improperly posed problems. 1980 MATHEMATICS SUBJECT CLASSIFICATION CODES: $35 R 30,35 R 25$.

1. INTRODUCTION.

A lot of authors have dealt the backward heat problem and considered equations of various kind. It is known that this problem is an improperly posed problem and the dependence of the solution as function of the initial data is an important aspect of it. The a priori inequalities (see Sigillito [1]) give immediately several informations. Among the methods of investigation, that of the logarithmic convexity is relatively simple when one is able to define - the difficulty is 
there - the functional which leads us to the required result (see, e.g. Knops [2]). But in the particular case of the hereafter equation, the functional is, for reasons of analogy, rather easy to determine.

2. EVALUATion of $\|u(x, t)\|$ AND $\left\|u_{t}(x, t)\right\|$ WITH $u \in C_{\alpha \beta}$.

Let us consider the backward heat equation:

$$
\begin{array}{rlrl}
\Delta u+u_{t} & =0 & \text { in } D \times[O, T) \\
u=0 & \text { on } \partial D \times[0, T)
\end{array}
$$

and the initial data $u(x, 0)=f(x)$

$D$ is a bounded open domain in $\mathbb{R}^{\mathrm{n}}$ with smooth boundary $\partial \mathrm{D}$.

Let $C_{\alpha \beta}$ be the class of the functions $\theta(x, t)$, continuous in $\bar{D} \times[0, T]$, so that

$\alpha$ ) for fixed $t \in(O, T), \theta$ is twice continuously differentiable in the $x$-variable and for $x \in D, \theta$ is continuously differentiable in $t$ $[t \in(O, T)]$

B) $\int_{D}\left|\frac{\partial}{\partial t} \theta(x, T)\right|^{2} d x \leq K^{2}$.

Then, if the solution $u$ of $(2.1-3)$ is subjected to belong to $C_{\alpha \beta}$, one has: $\quad \forall t \leqslant T, \quad\left\|u_{t}\right\| \leq k^{1 / T}\left\|u_{t}(x, 0)\right\|^{1-t / T}$

where, by definition $\|v(x, t)\|^{2}=\int_{D} v^{2}(x, t) d x$.

Let us set $g(x)=u_{t}(x, 0)$; then, the evaluation of $\left\|u_{t}\right\|$ is found if an upper bound of $\|\mathrm{g}(\mathrm{x})\|$ is known.

To prove (2.4), let us use the property of the logarithmic convexity applied to the functional (see [3], p. 11-12)

$$
\Psi(t)=\int_{D} u_{t}^{2}(x, t) d x
$$

One has: $\Psi^{\prime}(t)=2 \int_{D} u_{t} u_{t t} d x=-2 \int_{D} u_{t} \Delta u_{t} d x=-2 \Phi_{\partial D} u_{t} \cdot \frac{\partial}{\partial n} u_{t} d \sigma+$

$$
+2 \int_{D}\left|\operatorname{gradu}_{t}\right|^{2} \mathrm{dx}=2 \int_{\mathrm{D}}\left|\operatorname{gradu}_{t}\right|^{2} \mathrm{dx}
$$


since (2.2) implies that $\frac{\partial u}{\partial t}=0$ on $\partial D$ when $t \in(O, T)$. It follows that $\Psi "(t)=4 \int_{D} \operatorname{gradu} u_{t} \cdot g r a d u_{t t} d x=4 \Phi_{\partial D} u_{t t} \cdot \frac{\partial}{\partial n} u_{t} d \sigma-4 S_{D} u_{t t} \Delta u_{t} d x=$ $=4 \int_{D} u_{t t}^{2} d x$.

Using Schwarz's inequality one finds:

$$
\Psi \Psi^{\prime \prime}-\psi^{\prime 2} \geq 0 \quad \text { or } \quad(\ln \Psi) " \geq 0 \text {. }
$$

Thus, $\Psi \leq \Psi(T)^{t / T} \cdot \Psi(0)^{1-t / T}$, that is to say

$$
\left\|u_{t}\right\|^{2} \leq k^{2 t / T}\|g\|^{2(1-t / T)}
$$

On the other hand, one has:

$$
\delta_{D} u_{t} d x=-\delta_{D} u \Delta u d x=\delta_{D} \mid \text { gradu }\left.\right|^{2} d x \geq \lambda_{1} \delta_{D} u^{2} d x
$$

$$
\text { with } \left.\lambda_{1}=\text { first eigenvalue of the problem } \begin{array}{rl}
\Delta \phi+\lambda \phi & =0 \text { in } \mathrm{D} \\
\phi & =0 \text { on } \partial \mathrm{D}
\end{array}\right\}
$$

Then, for $t=T$

$\lambda_{1}^{2}\left(\delta_{D} u^{2} d x\right)^{2} \leqslant \delta_{D} u^{2} d x \cdot \delta_{D} u_{t}^{2} d x, \delta_{D} u^{2} d x \leqslant \frac{1}{\lambda_{1}^{2}} \delta_{D} u_{t}^{2} d x \leq\left(\frac{K}{\lambda_{1}}\right)^{2}=m^{2}$

and consequently (see [3], p. 13)

$$
\|u\| \leq\left(\frac{K}{\lambda_{1}}\right)^{t / T}\|f\|^{1-t / T}
$$

Now, let us substitute the condition $\beta$ ) of the class $C_{\alpha \beta}$ by

B') $\delta_{D}|\theta(x, t)|^{2} d x \leq K^{\prime 2}$

and let $C_{\alpha \beta^{\prime}}$, be the class of functions which satisfy $\alpha$ ) and $\left.\beta^{\prime}\right)$. Let us show why one can find one bound of $\left\|u_{t}\right\|$ if $u \in C_{\alpha \beta^{\prime}}$.

3. EVALUATiON OF $\left\|u_{t}(x, t)\right\|$ WITH $u \in C_{\alpha \beta^{\prime}}$.

LEMMA: if $u \in C_{\alpha \beta},\left\|u_{t}(x, t)\right\| \leqslant M_{\tau}$ for $t \leqslant T-\tau, 0<\tau \leqslant T$

PROOF : We remark first that $\Phi(t)=: \delta_{D} u^{2}(x, t) d x$ is an increasing function since $\Phi^{\prime}=2 \int_{D} u_{t} d x=2 \int_{D}|g r a d u|^{2} d x>0$. Thus,

$$
\Phi(t) \leq K^{\prime 2} \text { for } t \leq T
$$

Let $h(t)$ be a function of class $C^{2}$ and let us define 


$$
\begin{aligned}
& I=\int_{t=a}^{t=b} s_{D} h(t) u_{t}^{2}(x, t) d x d t=-\int_{a}^{b}\left[s_{D} u_{t} \Delta u d x\right] h(t) d t= \\
& =-\int_{a}^{b}\left[\Phi_{\partial D} u_{t} \cdot \frac{\partial u}{\partial n} d \sigma-s_{D} \text { gradu.gradu }{ }_{t} d x\right] h(t) d t= \\
& =\frac{1}{2} \int_{D}\left[\int_{a}^{b} h \frac{\partial}{\partial t}|\operatorname{gradu}|^{2} d t\right] d x=\frac{1}{2} \int_{D}\left(\left.h|\operatorname{gradu}|^{2}\right|_{t=a} ^{t=b}-\int_{a}^{b}|\operatorname{gradu}|^{2} h^{\prime} d t\right) d x \\
& =\frac{1}{2}\left[\delta_{D} h \mid \text { gradu }\left.\left.\right|^{2} d x\right|_{a} ^{b}\right]-\frac{1}{2} \int_{a}^{b} h^{\prime}\left(\int_{D}|\operatorname{gradu}|^{2} d x\right) d t=\frac{1}{2}[\ldots]- \\
& -\frac{1}{2} \int_{a}^{b} h^{\prime} \int_{D} \frac{1}{2} \frac{\partial}{\partial t} u^{2} d x d t=\frac{1}{2}[\ldots]-\left.\frac{1}{4} \int_{D} h^{\prime} u^{2} d x\right|_{t=a} ^{t=b}+\frac{1}{4} \int_{D} \int_{a}^{b} h^{\prime \prime} u^{2} d x d t \\
& I=\frac{1}{2}\left[h(b) \int_{D}|\operatorname{gradu}(x, b)|^{2} d x-h(a) \int_{D}|\operatorname{gradu}(x, a)|^{2} d x\right]- \\
& -\frac{1}{4}\left[h^{\prime}(b) \int_{D} u^{2}(x, b) d x-h^{\prime}(a) \int_{D} u^{2}(x, a) d x\right]+ \\
& +\frac{1}{4} \int_{D^{\prime}} \int_{a}^{b} h^{\prime \prime}(t) u^{2}(x, t) d x d t \text {. }
\end{aligned}
$$

Then, $\forall \mathrm{T}_{1}<\mathrm{T}$

$\int_{0}^{T_{1}} \int_{D} u_{t}^{2} d x d t \leq \int_{O}^{T_{1}} \int_{D} u_{t}^{2} d x d t+\int_{T_{1}}^{T} \int_{D} \frac{T-t}{T-T_{1}} u_{t}^{2} d x d t$,

and using (3.3) for each term of the sum, we obtain $\int_{0}^{T_{1} s_{D}} u_{t}^{2} d x d t \leq \frac{1}{2}\left[s_{D}\left|\operatorname{gradu}\left(x, T_{1}\right)\right|^{2} d x-s_{D}|\operatorname{gradu}(x, 0)|^{2} d x\right]+$ $+\frac{1}{2}\left[-\int_{D}\left|\operatorname{gradu}\left(\mathrm{x}, \mathrm{T}_{1}\right)\right|^{2} \mathrm{dx}\right]+\frac{1}{4} \cdot \frac{1}{\mathrm{~T}-\mathrm{T}_{1}}\left[\int_{\mathrm{D}} \mathrm{u}^{2}\left(\mathrm{x}, \mathrm{T}_{1}\right) \mathrm{dx}-\int_{\mathrm{D}} \mathrm{u}^{2}(\mathrm{x}, \mathrm{O}) \mathrm{dx}\right]$ $\leq \frac{1}{4\left(T-T_{1}\right)} \int_{D} u^{2}\left(x, T_{1}\right) d x \leq \frac{1}{4\left(T-T_{1}\right)} K^{\prime}=: M_{1}^{2}$ by (3.2).

Clearly, $\int_{O^{S} D}^{s} u_{t}^{2} d x d t$ is an increasing function of $s$. Thus,

$$
\int_{O}^{s} \delta_{D} u_{t}^{2} d x d t \leq M_{1}^{2} \quad \forall s \leq T_{1}<T
$$

Also,

$$
M_{1}^{2} \geq \int_{O}^{T_{1}} \int_{D} u_{t}^{2} d x d t \geq \int_{T_{0} \rho_{D}}^{T_{1}} u_{t}^{2} d x d t=\int_{T_{0}}^{T_{1}} \Psi(t) d t \quad\left(0 \leqslant T_{0}<T_{1}\right)
$$

But, $\Psi$ and $\Psi^{\prime}$ are positive; this leads to $\mathrm{M}_{1}^{2} \geqslant \Psi\left(\mathrm{T}_{0}\right)\left(\mathrm{T}_{1}-\mathrm{T}_{0}\right)$,

$$
\Psi\left(\mathrm{T}_{0}\right) \leq \mathrm{K}^{\prime} / 4\left(\mathrm{~T}-\mathrm{T}_{1}\right)\left(\mathrm{T}_{1}-\mathrm{T}_{0}\right) \text {. }
$$

Setting finally $\mathrm{T}_{0}=\mathrm{T}-\tau, \mathrm{T}_{1}=\mathrm{T}-\tau / 2$, one arrives to

$$
\Psi(t) \leq \Psi(T-\tau) \leq K^{\prime 2} / \tau^{2}=: M_{\tau}^{2}, t \leq T-\tau \quad \text { q.e.d. }
$$

So, we can evaluate $\|\Delta u\|$ with a bound of $\|u(x, T)\|$.

Let us write $T-\tau=T^{\prime}$ and $\left\|u_{t}\left(x, T^{\prime}\right)\right\| \leq M^{\prime}$. Then, if $u_{t t}$ exists, one finds similarly $\quad s_{D} u_{t t}^{2} d x \leq M^{\prime 2}, \forall t \leqslant T "$, since 


$$
\Delta\left(u_{t}\right)+\left(u_{t}\right)_{t}=0 \text { in } D \times[0, T) \text { and }\left(u_{t}\right)=0 \text { on } \partial D \times[O, T) \text {. }
$$

And generally:

$$
\int_{D}\left(\frac{\partial^{k} u}{\partial t^{k}}\right)^{2} d x \leq\left[M^{(k)}\right]^{2}, \quad t \leqslant T(k)<T, k \in \mathbb{N}
$$

REMARK: A result analogous to (3.1) can be obtained if we consider the serie $u(x, t)=\sum_{n=1}^{\infty} a_{n} u_{n}(x) e^{\lambda} n^{t}$ where $u_{n}$ are the eigenfunctions of (2.7), $\lambda_{n}$ the eigenvalues and $a_{n}$ the Fourier coefficients of $f(x)$. But, because of the necessity of the uniform convergence, the developments are long and rather complicated.

\section{ACKNOWLEDGMENT}

This work was supported by the Swiss National Science Foundation. I thank also cordially Professor L. E. Payne for his advice.

\section{REFERENCES}

[1] Sigillito, V.G., Explicit a priori inequalities with applications to boundary value problems, Research Notes in Mathematics 13, Pitman (1977).

[2] Knops, R.J., Logarithmic convexity and other techniques applied to problems in continuum mechanics, Lecture Notes in Mathematics 316, Springer Verlag (1973).

[3] Payne, L.E., Improperly posed problems in partial differential equations, Regional Conference Series in Applied Mathematics 22, Philadelphia (SIAM 1975). 


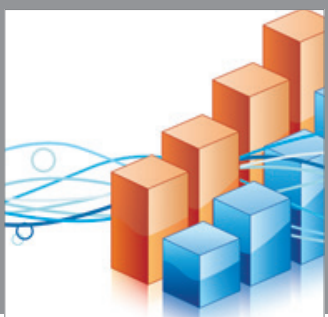

Advances in

Operations Research

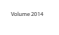

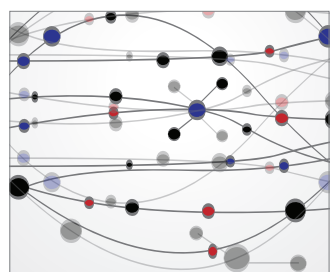

\section{The Scientific} World Journal
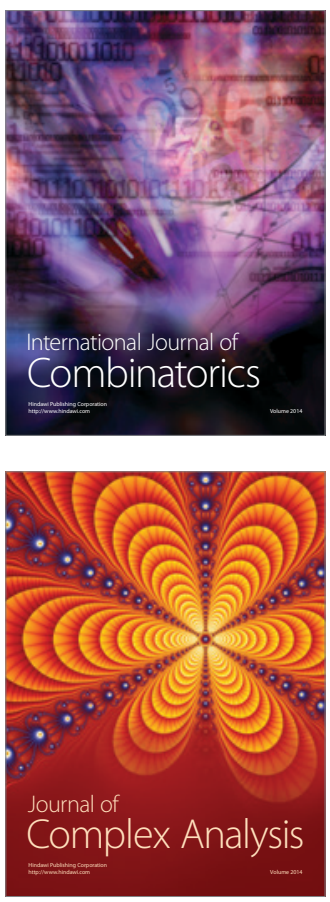

International Journal of

Mathematics and

Mathematical

Sciences
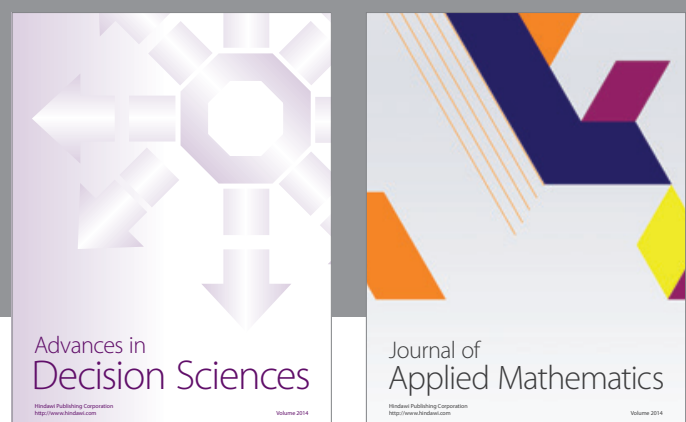

Journal of

Applied Mathematics
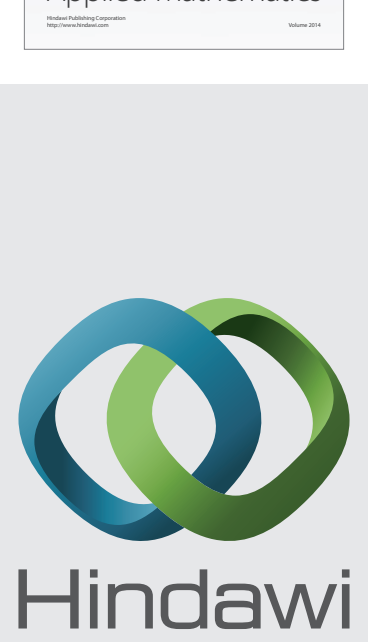

Submit your manuscripts at http://www.hindawi.com
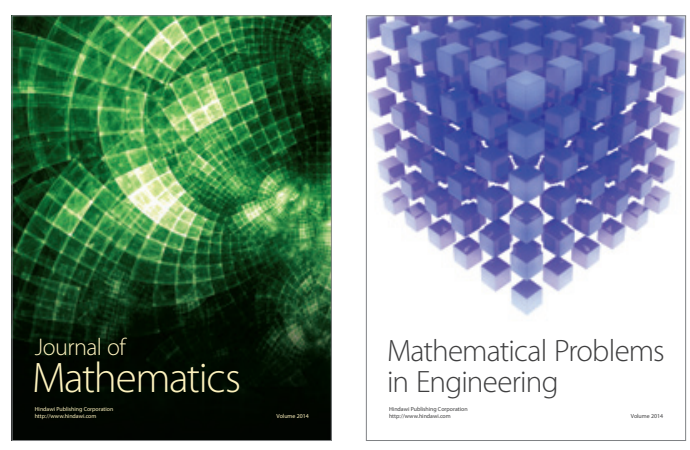

Mathematical Problems in Engineering
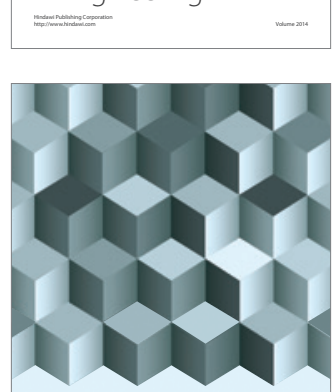

Journal of

Function Spaces
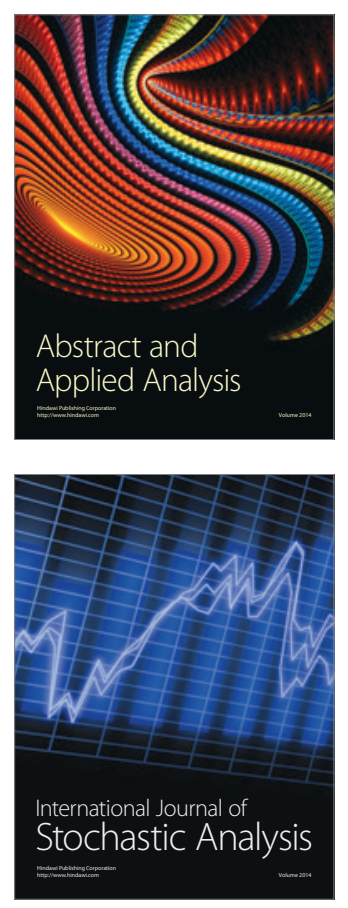

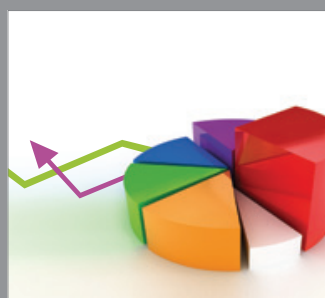

ournal of

Probability and Statistics

Promensencen
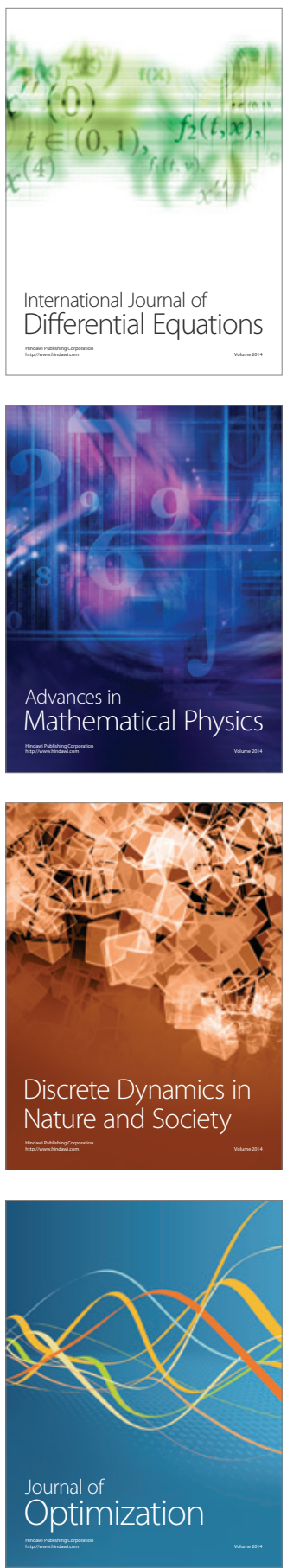